\begin{abstract}
Roman Bader ${ }^{1}$
Research School of Engineering,

The Australian National University,

Canberra ACT 2601, Australia

e-mail: roman.bader@anu.edu.au

Sophia Haussener ${ }^{1}$

Institute of Mechanical Engineering, Ecole Polytechnique Fédérale de Lausanne,

Lausanne 1015, Switzerland

e-mail: sophia.haussener@epfl.ch

\author{
Wojciech Lipiński ${ }^{1}$ \\ Research School of Engineering, \\ The Australian National University, \\ Canberra ACT 2601, Australia
}

e-mail: wojciech.lipinski@anu.edu.au

\section{Optical Design of Multisource High-Flux Solar Simulators}

We present a systematic approach to the design of a set of high-flux solar simulators (HFSSs) for solar thermal, thermochemical, and materials research. The generic simulator concept consists of an array of identical radiation modules arranged in concentric rows. Each module consists of a short-arc lamp coupled to a truncated ellipsoidal specular reflector. The positions of the radiation modules are obtained based on the rim angle, the number of concentric rows, the number of radiation modules in each row, the reflector radius, and a reflector spacing parameter. For a fixed array of radiation modules, the reflector shape is optimized with respect to the source-to-target radiation transfer efficiency. The resulting radiative flux distribution is analyzed on flat and hemispherical target surfaces using the Monte Carlo ray-tracing technique. An example design consists of 18 radiation modules arranged in two concentric rows. On a 60-mm dia. flat target area at the focal plane, the predicted radiative power and flux are $10.6 \mathrm{~kW}$ and $3.8 \mathrm{MW} \mathrm{m} \mathrm{m}^{-2}$, respectively, and the predicted peak flux is $9.5 \mathrm{MW} \mathrm{m}^{-2}$. [DOI: 10.1115/1.4028702]
\end{abstract}

Keywords: solar simulation, concentrated solar energy, high-flux, high-temperature

\section{Introduction}

Solar technologies that require point-focused concentrated sunlight, such as thermal receivers and solar reactors, can be tested under actual sunlight using solar furnaces [1,2], solar towers [3,4], and solar dishes [5,6], or with artificial concentrated sunlight using HFSSs. HFSSs are particularly useful for lab-scale $(\sim 1-10 \mathrm{~kW})$ prototype testing, as they provide a controlled, steady, and reproducible source of concentrated radiation, independent of the availability of direct sunlight. Furthermore, embedding the HFSS in an existing laboratory environment simplifies the logistics and provides a weather-protected indoor testing environment. HFSSs have been employed for several years in solar thermal and thermochemical research, and for high-temperature materials testing and processing. Table 1 compares the design and radiation characteristics of six HFSSs reported in literature. ${ }^{2}$ They consist of 1-10 radiation modules, each having an xenon or an argon arc lamp positioned at the focus of an elliptical specular reflector, either a point-focusing truncated ellipsoid of revolution or a line-focusing truncated elliptical trough. These HFSSs deliver between 0.9 and approx. $20 \mathrm{~kW}$ of radiative power onto a $60-\mathrm{mm}$ dia. target located at the focal plane, with average flux values ranging from 0.1 to $6.8 \mathrm{MW} \mathrm{m}^{-2}$. Peak fluxes range from 3.8 to $16 \mathrm{MW} \mathrm{m}^{-2}$. These radiation characteristics of the HFSSs are representative of the above mentioned point-focusing solar concentrators. In addition, the emission spectrum of a xenon arc lamp resembles that of blackbody radiation at $6000 \mathrm{~K}$ and hence approximates the terrestrial solar spectrum, as shown in Fig. 1.

Geometrical relations have been formulated to describe the design of HFSSs with seven lamps [14] and with ten lamps [12]. In this paper, we formulate the relations describing the geometry of a set of HFSSs. The generic simulator concept consists of an array of identical radiation modules arranged in concentric rows.

\footnotetext{
${ }^{1}$ Corresponding authors.

${ }^{2}$ Additional solar simulators have been mentioned in the literature, including a one-lamp design producing a peak radiative flux of $1.11 \mathrm{MW} \mathrm{m}^{-2}$ [7], a two-lamp design delivering a radiative flux of $2.15 \mathrm{MW} \mathrm{m} \mathrm{m}^{-2}$ over a $8 \mathrm{~mm}$ dia. target [8], and a seven-lamp design, which, in combination with a CPC, delivers $2.87 \mathrm{MW} \mathrm{m}^{-2}$ over a $40 \mathrm{~mm}$ dia. target [9].

Contributed by the Solar Energy Division of ASME for publication in the Journal of Solar ENERgy EngineERING: InCluding Wind ENERgy AND BuIlding Energy Conservation. Manuscript received March 31, 2014; final manuscript received September 10, 2014; published online October 23, 2014. Assoc. Editor: Dr Akiba Segal.
}

Each module consists of a short-arc lamp coupled to a truncated ellipsoidal mirror. The positions and orientations of the radiation modules are obtained based on the rim angle, the number concentric rows, number of radiation modules in each row, the reflector radius, and a reflector spacing parameter. Once the array of radiation modules has been determined, the eccentricity of the ellipsoidal reflector is optimized to maximize the source-to-target radiation transfer efficiency, taking into account the measured directional emission distribution of the lamp. A Monte Carlo raytracing model is formulated to analyze and optimize the HFSS design with respect to the radiative flux distribution at and behind the focal plane. An exemplary HFSS design is described, consisting of 18 radiation modules arranged in two concentric rows, and the Monte Carlo ray-tracing results for this design are presented.

\section{Geometry}

2.1 Overview. The geometry of the radiation module is shown in Fig. 2. It consists of a specular reflector in the shape of a section of an ellipsoid of revolution with a lip of thickness $t$, and a high-intensity lamp that approximates a point light source positioned at focus 1 of the ellipsoid. The reflector focuses the radiation emitted by the lamp onto focus 2 of the ellipsoid of revolution at the focal distance $2 c$ from focus 1 . Figure 3 illustrates the arrangement of the radiation modules comprising the solar simulator. The radiation modules are arranged in $m$ (row index $j=1, \ldots, m)$ concentric rows, with $n_{j}$ radiation modules in row $j$ (module index $k=1, \ldots, n_{j}$ ), such that the center of the truncation cross section of each reflector, $\mathrm{P}_{j, k}$, lies on the surface of a virtual sphere of radius $d_{\text {center }}$, and the foci 2 of the radiation modules form one common focus. A hole of projected radius $r_{\text {central }}$ is incorporated at the center of the simulator.

The geometry of the solar simulator is established in two steps. In the first step, the positions and orientations of the truncation cross sections (Fig. 3) of the reflectors are determined based on the following prescribed parameters: the rim angle, $\Phi$, the number of concentric rows of radiation modules, $m$, the number of radiation modules in each row, $n_{j}$, the inner radius of the reflector, $r_{\text {in }}$, and the thickness of the lip of the reflector, $t$. In the second step, the shape of the reflector and the positions of the lamps are determined. Two Cartesian coordinate systems are used for the description and modeling of the radiation modules. The global coordinate system $(x, y, z)$ is located at the common focus, with $z$-axis 
Table 1 Comparison of HFSSs reported in literature; the radiation characteristics are obtained at the focal plane of the HFSS with all lamps in operation

\begin{tabular}{|c|c|c|c|c|c|c|}
\hline Location & $\begin{array}{l}\text { LBL } \\
\text { U.S. }\end{array}$ & $\begin{array}{c}\text { ETHZ } \\
\text { Switzerland }\end{array}$ & $\begin{array}{c}\text { PSI } \\
\text { Switzerland }\end{array}$ & $\begin{array}{c}\text { DLR } \\
\text { Germany }\end{array}$ & $\begin{array}{l}\text { UMN } \\
\text { U.S. }\end{array}$ & $\begin{array}{c}\text { Texas A\&M } \\
\text { Qatar }\end{array}$ \\
\hline Number of radiation modules & 1 & 1 & 10 & 10 & 7 & 1 \\
\hline Rim angle (deg) & $n / a$ & 45 & 40.6 & $n / a$ & 37.7 & $n / a$ \\
\hline Reflector shape & ellipsoid & ell. trough & ellipsoid & ellipsoid & ellipsoid & ellipsoid \\
\hline Lamp type & $\mathrm{Xe}$ & $\mathrm{Ar}$ & $\mathrm{Xe}$ & $\mathrm{Xe}$ & $\mathrm{Xe}$ & $\mathrm{Xe}$ \\
\hline El. power per lamp $\left(\mathrm{kW}_{\mathrm{e}}\right)$ & $20 / 30$ & 200 & 15 & 6 & 6.5 & 7 \\
\hline Radiative power on $60 \mathrm{~mm}$ dia. target $(\mathrm{kW})$ & $n / a$ & $>6.73$ & 20 & $\sim 20$ & 9.2 & 0.9 \\
\hline $\begin{array}{l}\text { Average radiative flux on } 60 \mathrm{~mm} \text { dia. } \\
\text { target }\left(\mathrm{MW} \mathrm{m}^{-2}\right)\end{array}$ & $n / a$ & $>2.38$ & 6.8 & $>4.5$ & 3.24 & 0.1 \\
\hline Peak radiative flux $\left(\mathrm{MW} \mathrm{m}^{-2}\right)$ & 16 & $>4.25$ & $>11$ & $\sim 5.0$ & 7.9 & 3.8 \\
\hline Literature source & Reference [10] & Reference [11] & Reference [12] & Reference [13] & References [14] and [15] & Reference [16] \\
\hline
\end{tabular}

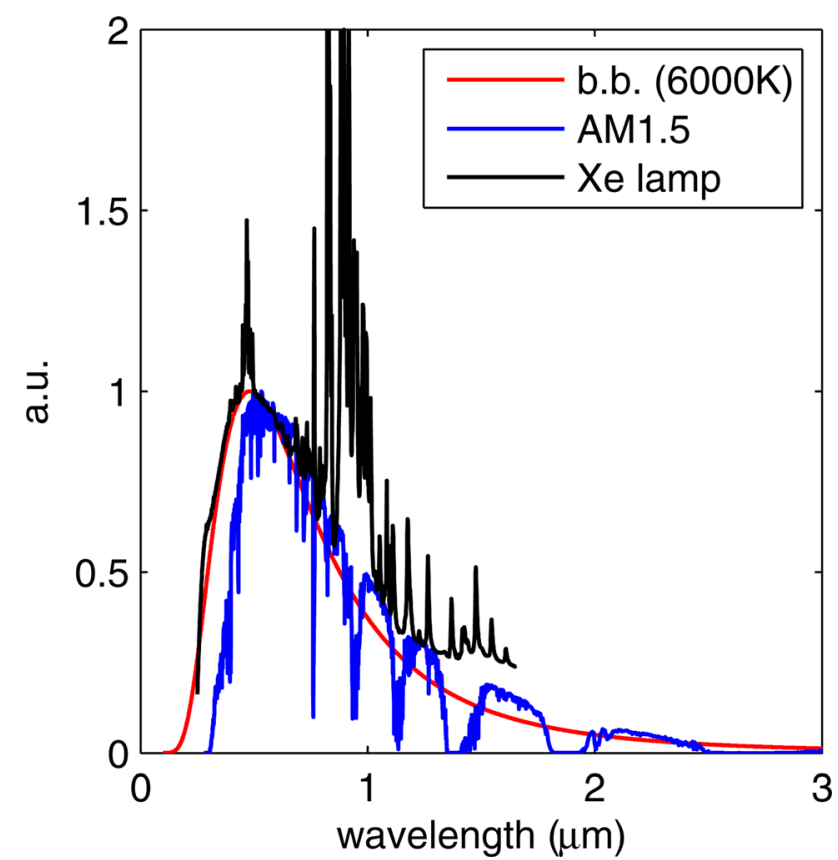

Fig. 1 Comparison of the emission spectrum of a xenon arc lamp (Osram XBO $6500 \mathrm{~W}$ OFR) [17] with the terrestrial solar spectrum (ASTM AM1.5) [18] and the emission spectrum of a blackbody at $6000 \mathrm{~K}$

pointing along the center axis of the simulator toward the reflectors, $y$-axis pointing in vertical direction, and $x$-axis direction obtained from the right-hand rule (Fig. 3). The unit vectors $\hat{\mathbf{i}}, \hat{\mathbf{j}}, \hat{\mathbf{k}}$ are defined to point along the global coordinate axes $x, y, z$, respectively. In addition, for each radiation module a local coordinate system $\left(x_{1}, y_{1}, z_{1}\right)$ is used, which is located at focus 1 of the radiation module, with $z$-axis direction along the axis of the radiation module toward focus 2 , and $x_{1}$ - and $y_{1}$-axis directions perpendicular to $z_{1}$ and to each other according to the right-hand rule. The unit vectors $\hat{\mathbf{i}}_{1}, \hat{\mathbf{j}}_{1}, \hat{\mathbf{k}}_{1}$ are defined to point along the local coordinate axes $x_{1}, y_{1}, z_{1}$, respectively (Fig. 2). The global coordinate system is used to describe the location and orientation of the radiation modules, while the local coordinate systems are used in the Monte Carlo ray-tracing model. The focal plane of the solar simulator is defined as the plane described by $z=0$.

2.2 Array of Radiation Modules. The geometrical relations described below by Eqs. (1)-(10) are used to determine the position and orientation of the truncation cross section of each reflector. For the outermost row of radiation modules, $j=m$, the parameter $l_{\text {clear }}$, defined as the horizontal distance between focal plane and inner edge of the closest reflector (Fig. 3(b)), is determined iteratively, such that neighboring reflectors touch each other at the outer edges of the reflector lips, as shown in Fig. $3(a)^{3}$. For rows $j=1, \ldots, m-1$, the parameter used for the same purpose is $\alpha_{\text {gap } . j}$, the angular spacing between adjacent lamp rows (Fig. 3(b)).

The distance $d_{\text {in }}$ between the inner edge of the reflector and the common focus of the simulator is given by

$$
d_{\text {in }}=\frac{l_{\text {clear }}}{\cos \Phi}
$$

The cone half-angle formed by the inner edge of the reflector and the axis of the radiation module, $\alpha_{\text {in }}$, is given by

$$
\alpha_{\text {in }}=\sin ^{-1}\left(\frac{r_{\text {in }}}{d_{\text {in }}}\right)
$$

The distance $d_{\text {center }}$ between the center of the truncation cross section of any of the reflectors in the array, $\mathrm{P}_{j, k}$, and the common focus of the simulator is given by

$$
d_{\text {center }}=\frac{r_{\text {in }}}{\tan \left(\alpha_{\text {in }}\right)}
$$

The truncation cross sections of the reflectors in row $j$ lie on a circle of radius $r_{\text {row }, j}$ (Fig. 3(b))

$$
r_{\text {row }, j}=d_{\text {center }} \sin \beta_{j}
$$

The angle $\beta_{j}$ describes the angle formed by the axis of a radiation module in row $j$ and the axis of the solar simulator. It is given by $\beta_{m}=\Phi-\alpha_{\text {in }}$ for row $j=m$, and by $\beta_{j}=\beta_{j+1}-2 \alpha_{\text {out }}-\alpha_{\text {gap }, j}$ for rows $j=1, \ldots, m-1$, where

$$
\alpha_{\text {out }}=\tan ^{-1}\left(\frac{r_{\text {out }}}{d_{\text {center }}}\right)
$$

with $r_{\text {out }}=r_{\text {in }}+t$. The angle $2 \chi_{j}$, the angular range occupied by one radiation module in row $j$, is found from the $2 \mathrm{D}$ geometry shown in Fig. 3(a). The projection of the truncation cross section of the reflector on the $x-y$ plane is an ellipse with semiprincipal axes $r_{\text {out }} \cos \beta_{j}$ and $r_{\text {out }}$.

Angle $\chi_{j}$ is formed by the direction $-\hat{\mathbf{i}}$ and the direction of the tangent to the ellipse that passes through $x=y=0$, described by the unit vector $\hat{\mathbf{t}}_{j}$. Hence

$$
\cos \chi_{j}=(-\hat{\mathbf{i}}) \cdot \hat{\mathbf{t}}_{j}
$$

${ }^{3}$ Setting $t$ to a slightly larger value than in reality introduces small gaps between the reflectors that facilitate the installation and adjustment of the radiation modules. 


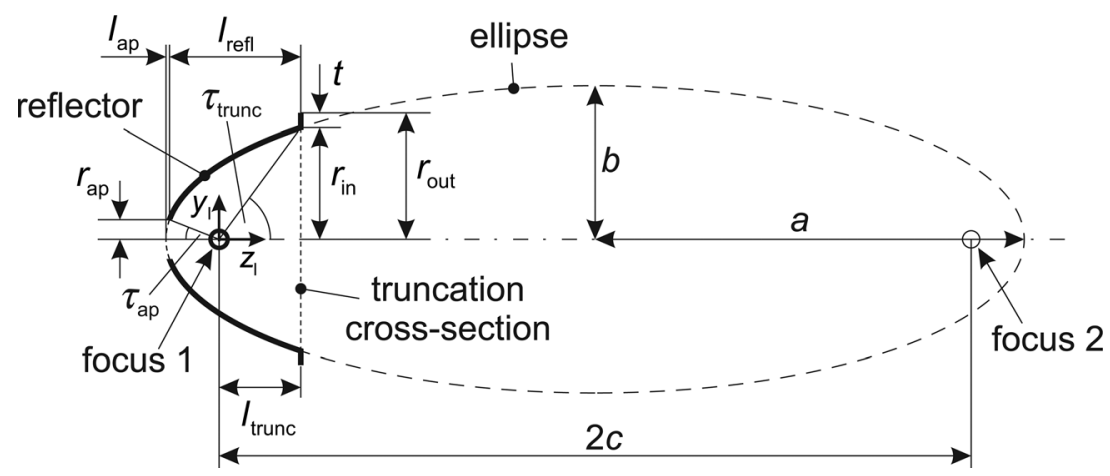

Fig. 2 Geometry of the radiation module consisting of an ellipsoidal reflector and a point light source (bold outlines); a hole of projected radius $r_{\text {ap }}$ at the apex of the reflector provides access for the lamp; the dashed-dotted line indicates the symmetry axis of the radiation module

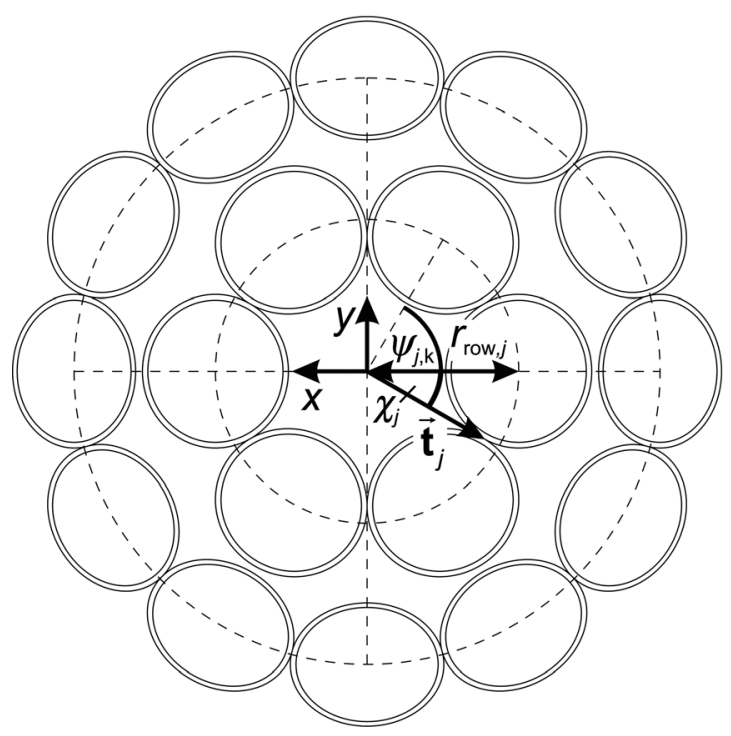

(a)

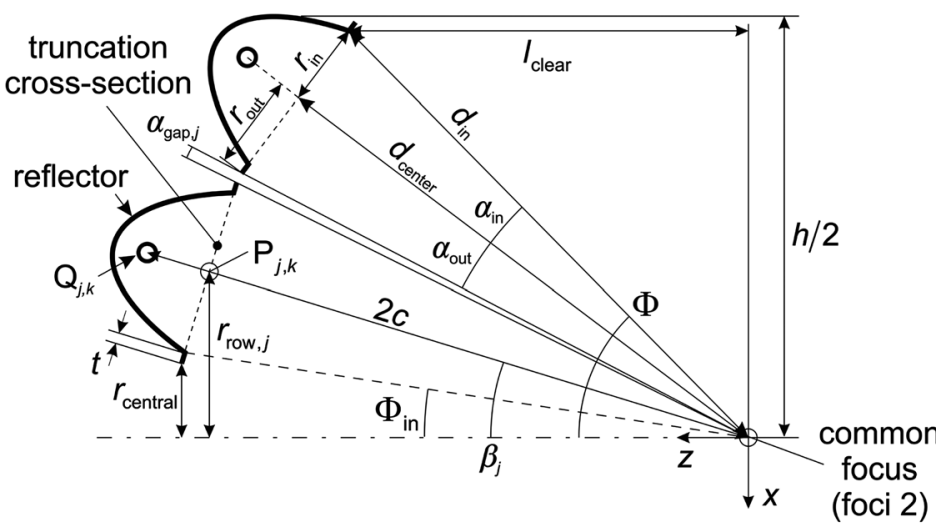

(b)

Fig. 3 Schematics of the solar simulator geometry: (a) projection of the truncation cross sections of the reflectors on the $x-y$ plane; (b) horizontal cross section at $y=0$, with the dashed-dotted line indicating the symmetry axis of the simulator

Unit vector $\hat{\mathbf{t}}_{j}$ is calculated from

$$
\begin{aligned}
\hat{\mathbf{t}}_{j} & =\left(\frac{t_{j, x}}{\sqrt{t_{j, x}^{2}+t_{j, y}^{2}}}, \frac{t_{j, y}}{\sqrt{t_{j, x}^{2}+t_{j, y}^{2}}}\right), \quad t_{j, x}=\frac{\left(r_{\text {out }} \cos \beta_{j}\right)^{2}}{r_{\text {row }, j}}-r_{\text {row }, j}, \\
t_{j, y} & =r_{\text {out }} \sqrt{1-\frac{\left(r_{\text {out }} \cos \beta_{j}\right)^{2}}{r_{\text {row }, j}^{2}}}
\end{aligned}
$$

The number of radiation modules in row $j$ is then obtained from

$$
n_{j}=\frac{\pi}{\chi_{j}}
$$

Equations (1)-(8) are iterated for each row, to determine $l_{\text {clear }}$ (for row $m$ ) and $\alpha_{\text {gap }, j}$ (for rows $j=1, \ldots, m-1$ ) such that the resulting value for $n_{j}$ obtained from Eq. (8) matches the prescribed number of lamps in row $j$.

\section{Journal of Solar Energy Engineering}

The center point of the truncation cross section of the $k$ th module in row $j$ is found from

$$
\mathrm{P}_{j, k}=d_{\text {center }}\left(\begin{array}{c}
-\sin \beta_{j} \cos \psi_{j, k} \\
\sin \beta_{j} \sin \psi_{j, k} \\
\cos \beta_{j}
\end{array}\right)
$$

with $\psi_{j, k}=2(k-1) \chi_{j}$, and the unit vector pointing along the axis of the radiation module toward the common focus is given by

$$
\hat{\mathbf{s}}_{j, k}=\frac{-\mathbf{P}_{\mathbf{j}, \mathbf{k}}}{\left|\mathbf{P}_{\mathbf{j}, \mathbf{k}}\right|}
$$

2.3 Geometry of Radiation Module. The parameter used to define the reflector shape and the position of the lamps is the eccentricity of the ellipsoid of revolution, defined as

$$
e=\sqrt{1-b^{2} / a^{2}}, \quad a \geq b
$$


where $a$ and $b$ are the semiprincipal axes of the ellipsoid of revolution (Fig. 2). The focal distance and the eccentricity are related via

$$
c=e a
$$

The distance between focus 1 and the truncation cross section is given as

$$
l_{\text {trunc }}=2 c-d_{\text {center }}
$$

A point on the inner edge of the reflector is described by

$$
\frac{r_{\text {in }}^{2}}{b^{2}}+\frac{\left(l_{\text {trunc }}-c\right)^{2}}{a^{2}}=1
$$

Solving the set of Eqs. (11)-(14) for $c$ yields

$$
c=\frac{d_{\text {center }}}{2}+\frac{1}{e^{2}-1}\left[\frac{d_{\text {center }}}{2}\left(1+e^{2}\right)-e \sqrt{d_{\text {center }}^{2}+r_{\text {in }}^{2}}\right]
$$

Backward substitutions yield the remaining unknowns $a, b$, and $l_{\text {trunc }}$. The eccentricity is determined iteratively to meet a prescribed truncation angle, $\tau_{\text {trunc }}$ (Fig. 2)

$$
\tau_{\text {trunc }}=\tan ^{-1}\left(\frac{r_{\text {in }}}{l_{\text {trunc }}}\right)
$$

Finally, the position of the lamps is found from

$$
\mathrm{Q}_{j, k}=2 c\left(\begin{array}{c}
-\sin \beta_{j} \cos \psi_{j, k} \\
\sin \beta_{j} \sin \psi_{j, k} \\
\cos \beta_{j}
\end{array}\right)
$$

Additional geometry parameters of interest are the reflector length

$$
l_{\text {refl }}=a-\left(c-l_{\text {trunc }}\right)-l_{\text {ap }}
$$

where $l_{\text {ap }}$ is the length of the truncated section at the apex of the reflector

$$
l_{\mathrm{ap}}=a\left(1-\sqrt{1-\left(r_{\mathrm{ap}} / b\right)^{2}}\right)
$$

and the inclination of the lamps relative to the horizontal position

$$
\gamma_{j, k}=\sin ^{-1}\left(s_{y, j, k}\right)
$$

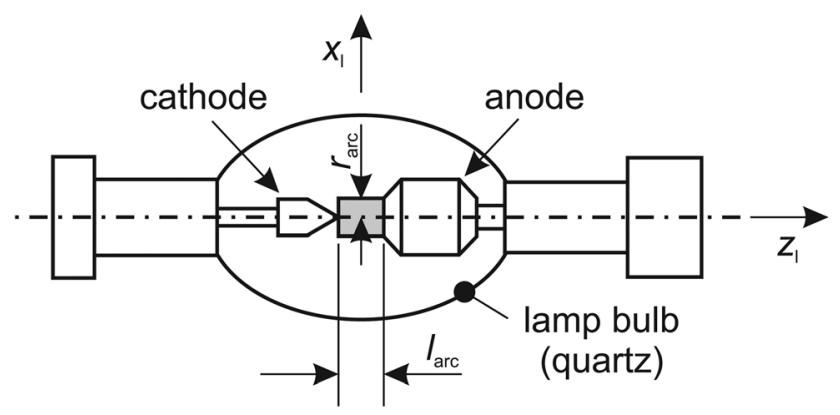

(a)
Two constraints are imposed on the design of the simulator. First, the reflector length should not exceed the longer semiprincipal axis

$$
l_{\text {refl }}+l_{\text {ap }}<a
$$

Second, the lamp position should be inside the reflector

$$
l_{\text {trunc }}>0
$$

\section{Optical Characterization}

A Monte Carlo ray-tracing simulation is formulated to predict the optical characteristics of the solar simulator [19]. The lamps are assumed to be xenon short-arc lamps. The lamp is modeled as a cylindrical emitting volume of length $l_{\text {arc }}$ and radius $r_{\text {arc }}$ (Fig. 4(a)), centered at focus 1 of the radiation module, with the cylinder axis aligned with the symmetry axis of the radiation module. Radiation is assumed to be emitted uniformly within the cylinder. For every ray, pseudorandom numbers on the interval $(0,1)$ are generated and used to determine the radial, axial, and circumferential coordinates of the emission point, and the azimuthal and polar angles describing the direction of emission of the ray. The angular emission distribution is given by the manufacturer in tabular form. It is characterized by a nearly uniform azimuthal distribution and the polar distribution shown in Fig. 4(b), with emission angles ranging from $19 \mathrm{deg}$ to $135 \mathrm{deg}$, measured relative to the $-z_{1}$ direction. The polar emission angle, $\theta$, is determined by equating the cumulative distribution function for $\theta$ to a random number

$$
\Re_{\theta}=\operatorname{CDF}(\theta)
$$

and determining $\theta$ from the tabular data with a binary search algorithm and linear interpolation.

Ray paths are tracked from the point of emission to the (last) target surface along the ray path. The reflector material is assumed to be polished alumina with specular reflectivity approximated by $\rho=0.9[21,22]$. Specular reflection on a surface with local unit surface normal vector $\hat{\mathbf{n}}$ is described by

$$
\hat{\mathbf{s}}_{\text {refl }}=\hat{\mathbf{s}}_{\text {inc }}-2\left(\hat{\mathbf{s}}_{\text {inc }} \cdot \hat{\mathbf{n}}\right) \hat{\mathbf{n}}
$$

where $\hat{\mathbf{s}}_{\text {inc }}$ and $\hat{\mathbf{s}}_{\text {refl }}$ describe the ray travel directions before and after the reflection, respectively. The ideal surface normal vector on the reflector is obtained from

$$
\hat{\mathbf{n}}_{\text {ideal }}=\frac{\left[\frac{\partial \mathbf{E}}{\partial \mathbf{x}_{1}}, \frac{\partial \mathbf{E}}{\partial \mathbf{y}_{1}}, \frac{\partial \mathbf{E}}{\partial \mathbf{z}_{1}}\right]}{\sqrt{\left(\frac{\partial \mathbf{E}}{\partial \mathbf{x}_{1}}\right)^{2}+\left(\frac{\partial \mathbf{E}}{\partial \mathbf{y}_{1}}\right)^{2}+\left(\frac{\partial \mathbf{E}}{\partial \mathbf{z}_{1}}\right)^{2}}}
$$

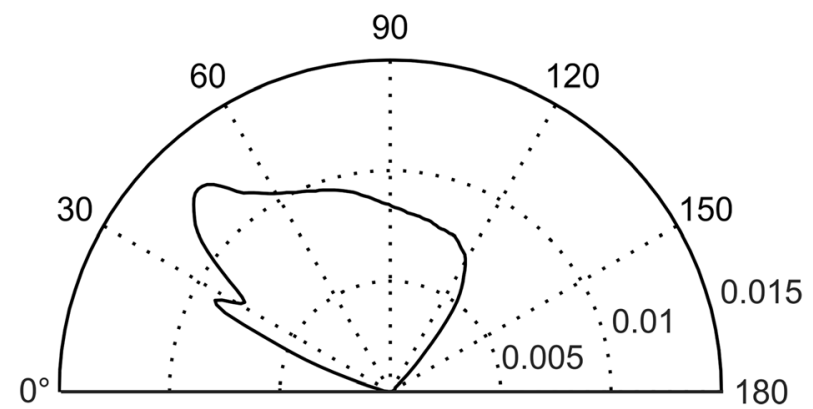

(b)

Fig. 4 Xenon short-arc lamp: (a) geometry: the gray area indicates the luminous area, modeled as a cylinder (adapted from Ref. [20]); (b) probability density function of the angular emission distribution of the lamp relative to the lamp axis (averaged circumferentially; $0 \mathrm{deg}$-pointing toward the apex of the reflector) 


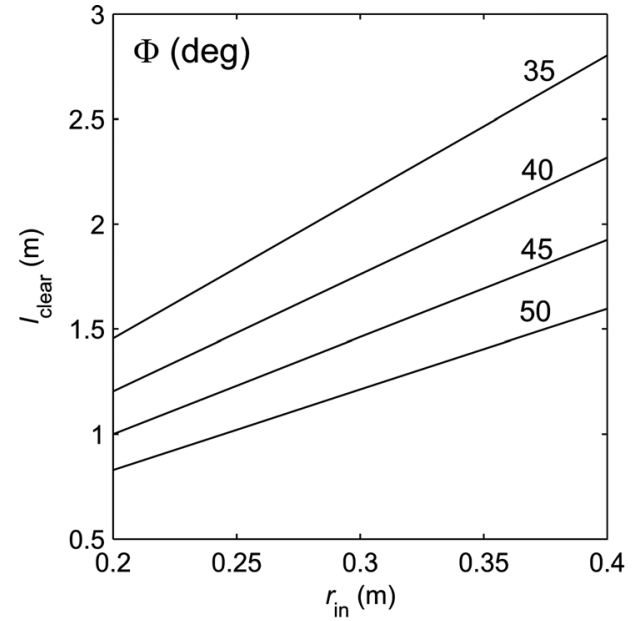

(a)

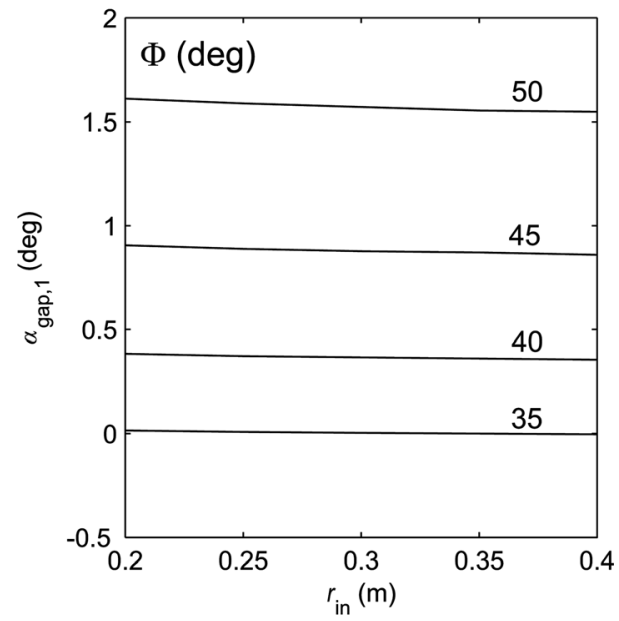

(b)

Fig. 5 Interrelation between geometry parameters for fixed $m=2, n_{j}=[6 ; 12]$, and $t=20 \mathrm{~mm}$, and variable $r_{\text {in }}$ and $\Phi:(a)$ minimal distance between HFSS and focal plane, $I_{\text {clear }},(b)$ angular spacing between the reflector rows, $\alpha_{\text {gap }, 1}$

where

$$
E=\frac{x_{1}^{2}}{b^{2}}+\frac{y_{1}^{2}}{b^{2}}+\frac{\left(z_{1}-c\right)^{2}}{a^{2}}-1=0
$$

describes the surface of the ellipsoid in local coordinates.

Different approaches have been used to describe reflector surface imperfections [23]. In all models it is assumed that the length scale of the surface error is much larger than the wavelength of the reflected radiation, and that surface errors are randomly distributed over the reflector surface. The surface error is introduced either by modifying the ideal surface normal vector, $\hat{\mathbf{n}}_{\text {ideal }}$, obtained from Eq. (25), or by modifying the reflected ray vector, $\hat{\mathbf{s}}_{\text {refl }}$, obtained from Eq. (24) with $\hat{\mathbf{n}}=\hat{\mathbf{n}}_{\text {ideal }}$. The modification of $\hat{\mathbf{n}}_{\text {ideal }}$ or $\hat{\mathbf{s}}_{\text {refl }}$ is described either with polar coordinates $(\varphi, \theta)$, or with two polar angles $\left(\theta_{1}, \theta_{2}\right)$ perpendicular to each other. When polar coordinates $(\varphi, \theta)$ are used, $\varphi$ is assumed to be uniformly distributed between 0 and $2 \pi$, while for $\theta$ either a Gaussian probability density function with standard deviation $\sigma_{1}$ (model 1a) [24] or a Rayleigh probability density function with mode $\theta_{\mathrm{m}}$ (model 1b) [25] is used. When two polar angles $\left(\theta_{1}, \theta_{2}\right)$ are used, both $\theta_{1}$ and $\theta_{2}$ are described by Gaussian probability density functions with equal standard deviation $\sigma_{2}$ (model 2) [25]. Johnston [25] showed that using model $1 \mathrm{~b}$ with mode $\theta_{\mathrm{m}}=\sigma_{2}$ to describe the difference between $\hat{\mathbf{n}}_{\text {ideal }}$ and $\hat{\mathbf{n}}$ is equivalent to using model 2 . Comparison with experimentally measured surface error data indicates that this modeling approach is suitable. Moreover, Krueger [23] illustrated that using model 1a is similar to using model 2, when $\sigma_{1}=\sqrt{2} \sigma_{2}$.

Here, model $1 \mathrm{~b}$ is used to describe the difference between $\hat{\mathbf{n}}_{\text {ideal }}$ and $\hat{\mathbf{n}}$. For each ray, $\theta$ is obtained from

$$
\theta=\sin ^{-1}\left(\sqrt{-2 \theta_{\mathrm{m}}^{2} \ln \left(1-\Re_{\theta}\right)}\right)
$$

where $\Re_{\theta}$ denotes a random number between 0 and 1 . The mode of the Rayleigh distribution is assumed to be $\theta_{\mathrm{m}}=2.5 \mathrm{mrad}$. This value was determined by Krueger [23] for the type of reflectors assumed to be used here, based on a comparison of measured and simulated radiative power distributions at the focal plane. The azimuthal angle $\varphi$ is found from

$$
\varphi=2 \pi \Re_{\varphi}
$$

\section{Results and Discussion}

4.1 Geometrical Considerations. Equations (1)-(8), together with the design constraints defined in Sec. 2 (radiation modules arranged in concentric rows over a spherical surface; adjacent reflectors in each row), couple the prescribed parameters defined in Sec. $2, \Phi, m, n_{j}, r_{\text {in }}, t$, with the unknown parameters $l_{\text {clear }}$ and $\alpha_{\mathrm{gap}, j}$. Figure 5 shows the variation of $l_{\text {clear }}$ and $\alpha_{\mathrm{gap}, 1}$ as functions of $r_{\text {in }}$ and $\Phi$, for fixed $m=2, n_{j}=[6 ; 12]$, and $t=20 \mathrm{~mm}$. It shows that both increasing $l_{\text {clear }}$ and increasing $\Phi$ require an increase in the reflector radius, while $\alpha_{\text {gap }, 1}$ remains relatively small and nearly independent of the reflector radius. The upper

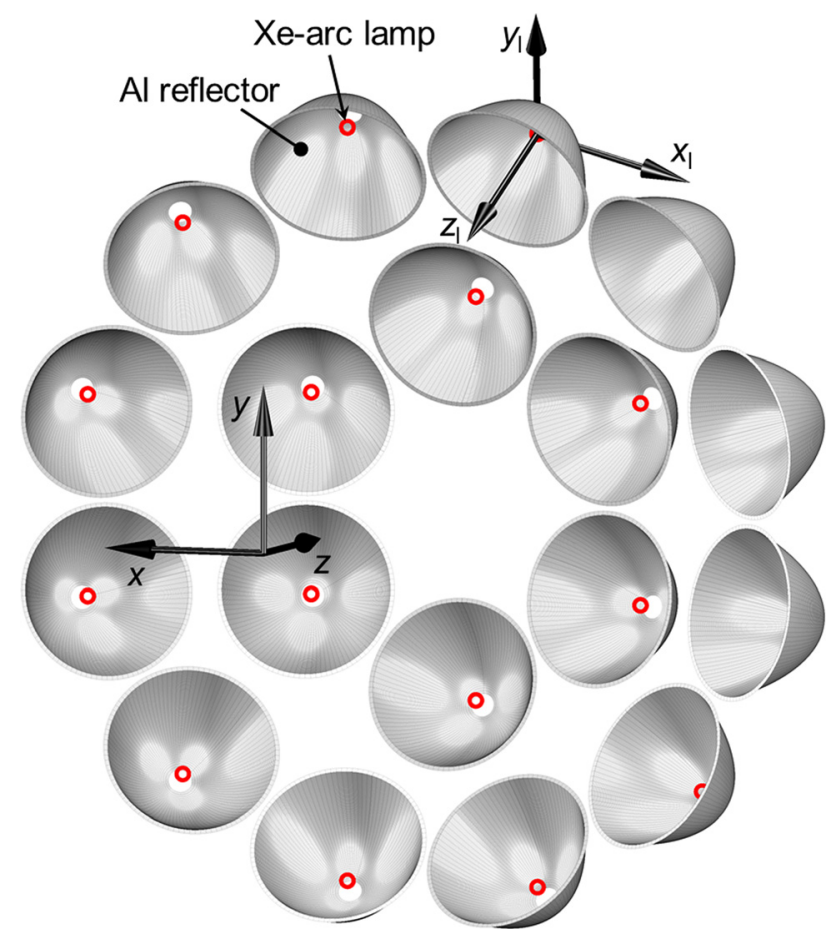

Fig. $63 \mathrm{D}$ rendering of the selected solar simulator geometry with the parameters listed in Table 2; circles (red) in the 18 reflector foci indicate the lamp positions 
Table 2 Geometrical parameters of the selected solar simulator

\begin{tabular}{lc}
\hline \hline Rim angle, $\Phi(\mathrm{deg})$ & 45 \\
Number of rows, $m$ & 2 \\
Number of radiation modules in row $j, n_{j}$ & 6,12 \\
Reflector radius, $r_{\text {in }}(\mathrm{mm})$ & 250.0 \\
Design lip thickness, $t(\mathrm{~mm})$ & 20.00 \\
Actual lip thickness $(\mathrm{mm})$ & 12.50 \\
View angle, $\tau_{\text {trunc }}(\mathrm{deg})$ & 50.00 \\
Clearance, $l_{\text {clear }}(\mathrm{mm})$ & 1231 \\
Height of solar simulator, $h(\mathrm{~m})$ & 2.53 \\
Radius of central hole, $r_{\text {central }}(\mathrm{mm})$ & 276.8 \\
Focal distance, $2 c(\mathrm{~mm})$ & 1933 \\
Reflector length, $l_{\text {refl }}(\mathrm{mm})$ & 272.4 \\
Max. lamp tilt angle in each row, $\gamma_{j, \mathrm{max}}(\mathrm{deg})$ & $18.04,35.30$ \\
Inner rim angle, $\Phi_{\text {in }}(\mathrm{deg})$ & 9.782 \\
Radius of apex hole, $r_{\text {ap }}(\mathrm{mm})$ & 35.00 \\
Apex view angle, $\tau_{\text {ap }}(\mathrm{deg})$ & 29.21 \\
Length of luminous area, $l_{\text {arc }}(\mathrm{mm})$ & 4.500 \\
Radius of luminous area, $r_{\text {arc }}(\mathrm{mm})$ & 0.750 \\
\hline
\end{tabular}

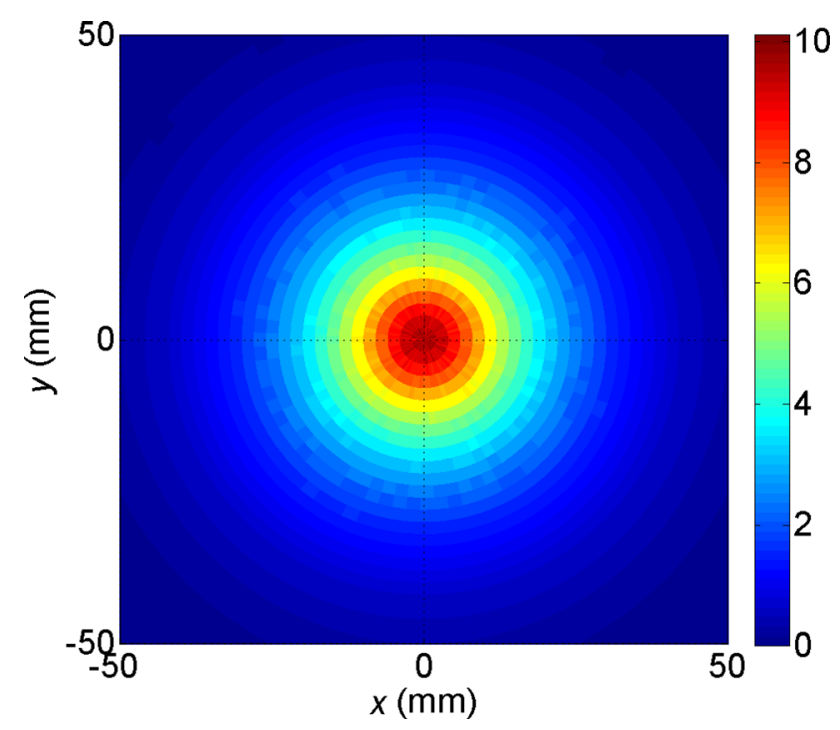

Fig. 7 Radiative flux distribution at the focal plane (in

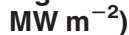

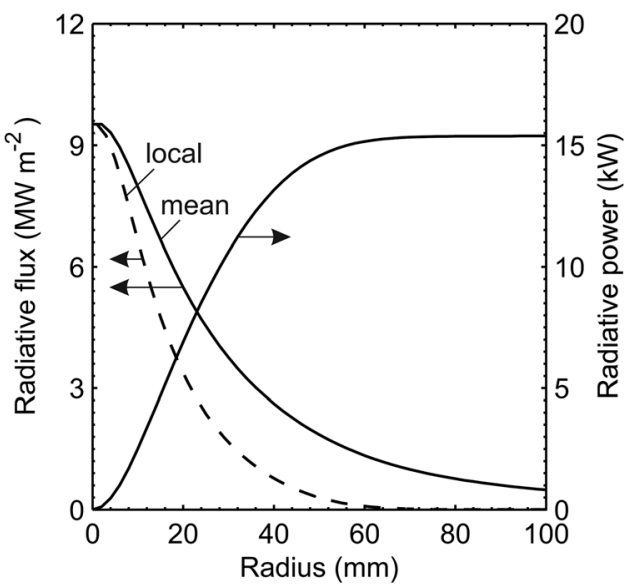

(a) limit for the reflector radius depends on the limitations imposed by the manufacturing process of the reflector. In addition, larger reflectors are heavier and tend to require thicker walls to achieve the same shape stability as smaller ones. Moreover, increasing $l_{\text {clear }}$ or $\Phi$ leads to an increase of the height of the simulator, $h$ (Fig. 3(b)). In practice, both $h$ and $l_{\text {clear }}$ are limited by the space available for the solar simulator. $l_{\text {clear }}$ should be large enough to: (i) install a safety shutter between the simulator and the focal plane (where the experiment is placed) and (ii) provide sufficient clearance between the simulator and the experiment to avoid any damage to the simulator due to the spillage of fluids, excessive heat, or flying parts in the event of failure of the experimental setup. Rim angles of solar dish concentrators usually are $>45$ deg for practical reasons. Hence, solar simulators with $\Phi<45 \mathrm{deg}$ are not representative of real solar dish concentrators and should be avoided.

4.2 Selected Geometry. Selecting $m=2, n_{j}=[6 ; 12]$, and $t=20 \mathrm{~mm}$, as in the example of Sec. 4.1, as well as $\Phi=45 \mathrm{deg}$, $r_{\text {in }}=0.25 \mathrm{~m}$, and $e=0.935$ (resulting in $\tau_{\text {trunc }}=50 \mathrm{deg}$ ), and rotating the inner and outer rows by $30 \mathrm{deg}$ and $15 \mathrm{deg}$, respectively, yields the simulator geometry shown in Fig. 6. The geometry parameters are listed in Table 2 .

The rim angle of $45 \mathrm{deg}$ corresponds to the rim angle that maximizes the solar concentration ratio at the focal plane of a solar dish concentrator [5]. Increasing the number of radiation modules influences several design aspects. It increases the flexibility at selecting the radiative flux and power at the focal plane. On the other hand, it also increases the complexity of the solar simulator, as each lamp requires a separate rectifier, cooling fan, and connections. Finally, a larger number of smaller lamps with lower power reduces the flux peaks on target surfaces behind the focal plane, which reduces the risk of "hot-spots" that can lead to damage of the tested solar device. The obtained clearance, $l_{\text {clear }}$, is sufficiently large to install a safety shutter between simulator and focal plane. The view angle, $\tau_{\text {trunc }}$, has been selected such that nearly all radiation emitted by the lamp in forward direction is intercepted by the reflector (Fig. 4(b)).

4.3 Optical Performance. The Monte Carlo ray-tracing simulation results for the simulator configuration described in Sec. 4.2 with all 18 lamps in operation are shown in Figs. 7-9. The simulation parameters are listed in Table 3. New lamps convert approx. $60 \%$ of the electric power input to radiation that is emitted from the Xe arc, the rest being lost as heat via convection and IR radiation [20]. Due to a degradation of the lamp electrodes, the radiative power emitted by the lamp decreases over its

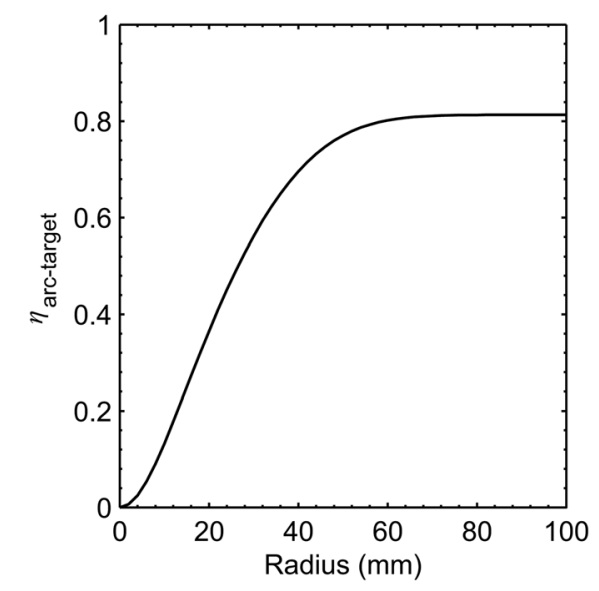

(b)

Fig. 8 (a) Local radiative flux, mean radiative flux, and radiative power as functions of the radial coordinate from the center of the focal plane; $(b)$ radiation transfer efficiency from the lamps to the focal plane as a function of the radial coordinate 


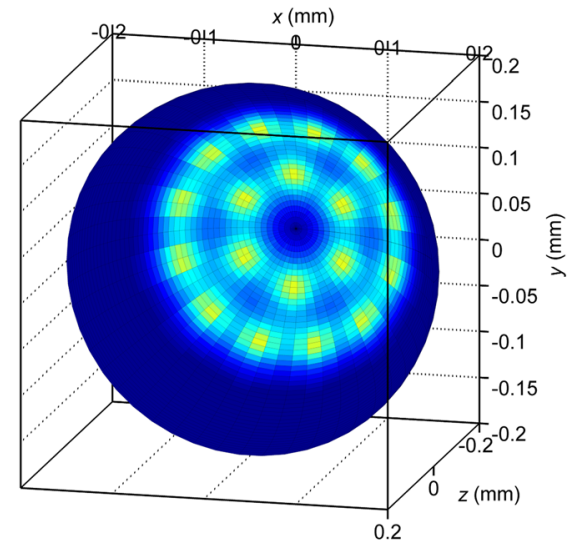

(a)

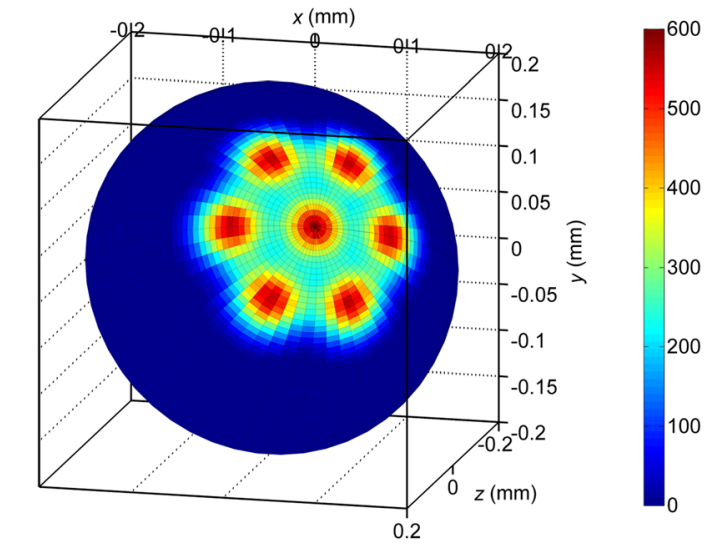

(b)

Fig. 9 Radiative flux distribution on a hemispherical target surface of $0.2 \mathrm{~m}$ radius, with its base at the focal plane and the hemisphere extending behind the focal plane, for (a) the 18-lamp HFSS design presented in this paper and $(b)$ the seven-lamp HFSS design with geometrical parameters listed in Table 5, which is similar to the design reported by Krueger et al. [14] (units of

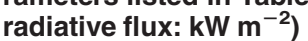

\section{Table 3 Simulation parameters}

Rated electric power input to the lamp (kW)

Factor accounting for part-load operation of the lamp

Electric-to-radiation conversion efficiency of the lamp [20]

Reflector surface error, $\theta_{\mathrm{m}}$ (mrad)

Specular reflectivity of reflector, $\rho[21,22]$

Table 4 Predicted radiative characteristics at the focal plane of the solar simulator with all 18 lamps in operation

\begin{tabular}{lcc}
\hline \hline Total radiative power $(\mathrm{kW})$ & 15.4 & \\
Peak radiative flux $\left(\mathrm{MW} \mathrm{m}^{-2}\right)$ & \multicolumn{2}{c}{} \\
\hline Target diameter $(\mathrm{mm})$ & 30 & 60 \\
\hline Radiative power $(\mathrm{kW})$ & 4.7 & 10.6 \\
Mean radiative flux $\left(\mathrm{MW} \mathrm{m}{ }^{-2}\right)$ & 6.7 & 3.8 \\
Radiation transfer efficiency $(\%)$ & 25 & 56 \\
\hline \hline
\end{tabular}

Table 5 Geometrical parameters of the seven-lamp solar simulator design used to assess the radiative flux distribution on a hemispherical target. The geometry is similar to that reported by Krueger et al. [14].

\begin{tabular}{lc}
\hline \hline Rim angle, $\Phi(\mathrm{deg})$ & 37.7 \\
Number of rows, $m$ & 2 \\
Number of radiation modules in row $j, n_{j}$ & 1,6 \\
Reflector radius, $r_{\text {in }}(\mathrm{mm})$ & 375.0 \\
Design lip thickness, $t(\mathrm{~mm})$ & 22.50 \\
View angle, $\tau_{\text {trunc }}(\mathrm{deg})$ & 58.40 \\
Clearance, $l_{\text {clear }}(\mathrm{mm})$ & 1441.0 \\
Focal distance, $2 c(\mathrm{~mm})$ & 2013.0 \\
Reflector length, $l_{\text {refl }}(\mathrm{mm})$ & 352.4 \\
Radius of apex hole, $r_{\text {ap }}(\mathrm{mm})$ & 35.00 \\
Length of luminous area, $l_{\text {arc }}(\mathrm{mm})$ & 6.300 \\
Radius of luminous area, $r_{\text {arc }}(\mathrm{mm})$ & 2.000 \\
\hline
\end{tabular}

lifetime. To provide some space to compensate for this reduction of the radiative power emitted by the lamp, the solar simulator is designed with the lamps operating at $70 \%$ of their nominal power.

The radiative flux distribution at the focal plane (Fig. 7) is axisymmetric and the results can be analyzed in 1D (radial direction), as shown in Fig. 8. Numerical results for selected target radii are listed in Table 4.
To analyze the angular uniformity of the radiation emitted by the solar simulator, the flux distribution is determined on a hemispherical target of $0.2 \mathrm{~m}$ radius, with its base at the focal plane and the hemisphere extending behind the focal plane. The result for the 18-lamp design with the parameters listed in Tables 2 and 3 is shown in Fig. 9(a). To assess the benefit of using an increased number of smaller radiation modules compared to a smaller number of larger modules, the result is compared to that obtained for a sevenlamp design, with the geometrical parameters listed in Table 5. The simulation parameters for the seven-lamp design are those in Table 3 , except for the rated lamp power, which is increased to $6.5 \mathrm{~kW}$. The result for the seven-lamp design is shown in Fig. 9(b).

The radiative power delivered onto the hemispherical target is similar for the two HFSS designs (18-lamp design: 15.4kW; sevenlamp design: $16.0 \mathrm{~kW}$ ). However, due to the lower power per lamp, the peak flux values are nearly $50 \%$ lower with the 18 -lamp design, and the flux spots created by the lamps are smaller in size. This reduces the risk of "hot-spots" and damage of tested solar devices. A strong correlation between the mechanical stress distribution in the wall of a cylindrical cavity receiver and the temperature distribution in the wall was shown by Hischier et al. [26]. The effect of the larger rim angle of the 18-lamp design ( $45 \mathrm{deg}$ as opposed to $37.7 \mathrm{deg}$ ) is also visible in Fig. 9, which leads to the distribution of the incident radiation over a larger area of the hemispherical target.

\section{Summary and Conclusions}

A systematic approach has been developed for the design of a set of HFSSs. The design is based on concentric rows of identical radiation modules that are arranged over a virtual sphere to form a common focus. The rim angle, the number of concentric rows, the number of radiation modules in each row, the reflector radius, and the reflector spacing are the input parameters, while the clearance between the simulator and the focal plane and the angular spacing between the concentric rows are the dependent parameters. This design method can be applied for the design of HFSSs with various rim angles, overall dimensions, number of radiation modules, and radiative power outputs.

A simulator design consisting of 18 radiation modules arranged in two concentric rows has been presented and characterized with the Monte Carlo ray-tracing method. The simulator delivers up to $15.4 \mathrm{~kW}$ of radiative power to the focal plane with a transfer efficiency of up to $81 \%$. The peak radiative flux is $9.5 \mathrm{MW} \mathrm{m}^{-2}$. On a $60-\mathrm{mm}$-dia. target area, the intercepted radiative power is $10.6 \mathrm{~kW}$, the mean flux is $3.8 \mathrm{MW} \mathrm{m} \mathrm{m}^{-2}$, and the transfer efficiency is $56 \%$. Comparing the radiative flux distribution on a 
0.2-m-radius hemispherical target obtained with the 18-lamp simulator design with that obtained with a seven-lamp design reveals a significant improvement in the angular uniformity of the flux distribution and a reduction of the peak flux values by nearly $50 \%$. This reduces the risk of hot-spots and material damage in the tested solar device.

\section{Acknowledgment}

We thank Lutz Schmidt and Christoph Baur of Kinoton GmbH for fruitful discussions and for providing important information on the manufacturability and technical data for the reflectors and light sources. The preliminary ray-tracing analyses of the simulator by Daryl Lee (University of Minnesota) and Valentin Fleury (EPFL) are gratefully acknowledged.

\section{Nomenclature}

$a=$ semimajor axes of the ellipsoidal reflector (m)

$b=$ semiminor axis of the ellipsoidal reflector $(\mathrm{m})$

$c=$ half of the focal distance $(\mathrm{m})$

$\operatorname{CDF}(\theta)=$ cumulative distribution function for variable $\theta$

$d_{\text {center }}=$ distance between center of truncation cross section and focus $(\mathrm{m})$

$d_{\text {in }}=$ distance between inner rim of reflector and focus (m)

$e=$ eccentricity of the ellipsoidal reflector

$E=$ function describing reflector surface

$h=$ height of solar simulator (m)

$\hat{\mathbf{i}}, \hat{\mathbf{j}}, \hat{\mathbf{k}}=$ unit vectors along the axes of the global coordinate system expressed in global coordinates

$\hat{\mathbf{i}}_{l}, \hat{\mathbf{j}}_{l}, \hat{\mathbf{k}}_{l}=$ unit vectors along the axes of the local coordinate system expressed in global coordinates

$j=$ module row index

$k=$ radiation module index

$l_{\text {ap }}=$ length of truncated section at apex of reflector $(\mathrm{m})$

$l_{\text {arc }}=$ length of luminous area $(\mathrm{m})$

$l_{\text {clear }}=$ minimal distance between HFSS and focal plane $(\mathrm{m})$

$l_{\text {refl }}=$ reflector length $(\mathrm{m})$

$l_{\text {trunc }}=$ distance between focus 1 and truncation cross section (m)

$m=$ number of module rows

$\hat{\mathbf{n}}=$ unit surface normal vector

$n_{j}=$ number of lamps in row $j$

$\mathrm{P}_{j, k}=$ center of truncation cross section of reflector $k$ in row $j$

$\mathbf{P}_{\mathbf{j}, \mathbf{k}}=$ position vector to center of truncation cross section of reflector $k$ in row $j$

$\mathrm{Q}_{j, k}=$ lamp position

$\Re=$ pseudo random number

$r_{\text {ap }}=$ radius of apex hole $(\mathrm{m})$

$r_{\mathrm{arc}}=$ radius of luminous area $(\mathrm{m})$

$r_{\text {central }}=$ radius of central hole $(\mathrm{m})$

$r_{\text {in }}=$ inner radius of reflector $(\mathrm{m})$

$r_{\text {out }}=$ outer radius of reflector $(\mathrm{m})$

$r_{\text {row }, j}=$ radial position of radiation modules in row $j(\mathrm{rad})$

$\hat{\mathbf{s}}=$ unit vector along ray travel direction

$\hat{\mathbf{s}}_{j, k}=$ unit vector along axis of radiation module $k$ in row $j$

$t=$ reflector lip thickness $(\mathrm{m})$

$\mathbf{t}_{j}=$ tangential vector to reflector

$x, y, z=$ global Cartesian coordinates

$x_{1}, y_{1}, z_{1}=$ local Cartesian coordinates

\section{Greek Symbols}

$\alpha_{\text {gap }, j}=$ angular spacing of reflector rows ( $\left.\mathrm{rad}\right)$

$\alpha_{\text {in }}=$ half cone angle subtended by reflector inner edge (rad)

$\alpha_{\text {out }}=$ half cone angle subtended by reflector outer edge $(\mathrm{rad})$ $\beta_{j}=$ angular position of radiation modules in row $j(\mathrm{rad})$

$\gamma=$ tilt angle of the lamp axis (rad)

$\eta_{\text {arc-target }}=$ radiation transfer efficiency

$\theta=$ cone angle in polar coordinates $(\mathrm{rad})$

$\theta_{\mathrm{m}}=$ mode of Rayleigh probability density function (mrad)

$\chi_{j}=$ half-angular extent occupied by radiation module in row $j(\mathrm{rad})$

$\rho=$ specular reflectivity

$\sigma=$ standard deviation ( $\mathrm{mrad})$

$\tau_{\text {ap }}=$ apex view angle (rad)

$\tau_{\text {trunc }}=$ view angle $(\mathrm{rad})$

$\varphi=$ azimuthal angle in polar coordinates $(\mathrm{rad})$

$\Phi=$ rim angle of simulator $(\mathrm{deg})$

$\Phi_{\text {in }}=$ inner rim angle of simulator (deg)

$\psi_{j, k}=$ angular position of radiation module $k$ in row $j(\mathrm{rad})$

\section{Subscripts}

inc $=$ incident

refl $=$ reflected

\section{Abbreviations}

$\mathrm{AM}=$ air mass

HFSS $=$ high-flux solar simulator

\section{References}

[1] Ries, H., and Schubnell, M., 1990, "The Optics of a Two-Stage Solar Furnace," Sol. Energy Mater., 21(2-3), pp. 213-217.

[2] Haueter, P., Seitz, T., and Steinfeld, A., 1999, “A New High-Flux Solar Furnace for High-Temperature Thermochemical Research,” ASME J. Sol. Energy Eng., 121(1), pp. 77-80.

[3] Hildebrandt, A. F., and Vant-Hull, L. L., 1977, "Power With Heliostats," Science, 197(4309), pp. 1139-1146.

[4] Radosevich, L. G., and Skinrood, A. C., 1989, "The Power Production Operation of Solar One, the 10 MWe Solar Thermal Central Receiver Pilot Plant," ASME J. Sol. Energy Eng., 111(2), pp. 144-151.

[5] Rabl, A., 1976, "Comparison of Solar Concentrators," Sol. Energy, 18(2), pp. 93-111.

[6] Lovegrove, K., Burgess, G., and Pye, J., 2011, "A New $500 \mathrm{~m}^{2}$ Paraboloidal Dish Solar Concentrator," Sol. Energy, 85(4), pp. 620-626.

[7] Gokon, N., Takahashi, S., Yamamoto, H., and Kodama, T., 2008, "Thermochemical Two-Step Water-Splitting Reactor With Internally Circulating Fluidized Bed for Thermal Reduction of Ferrite Particles," Int. J. Hydrogen Energy, 33(9), pp. 2189-2199.

[8] Tamaura, Y., and Kaneko, H., 2005, "Oxygen-Releasing Step of $\mathrm{ZnFe}_{2} \mathrm{O}_{4}$ $\left(\mathrm{ZnO}+\mathrm{Fe}_{3} \mathrm{O}_{4}\right)$-System in Air Using Concentrated Solar Energy for Solar Hydrogen Production," Sol. Energy, 78(5), pp. 616-622.

[9] Furler, P., Scheffe, J. R., and Steinfeld, A., 2012, "Syngas Production by Simultaneous Splitting of $\mathrm{H}_{2} \mathrm{O}$ and $\mathrm{CO}_{2}$ Via Ceria Redox Reactions in a HighTemperature Solar Reactor," Energy Environ. Sci., 5(3), pp. 6098-6103.

[10] Kuhn, P., and Hunt, A., 1991, "A New Solar Simulator to Study High Temperature Solid-State Reactions With Highly Concentrated Radiation," Sol. Energy Mater., 24(1-4), pp. 742-750.

[11] Hirsch, D., Zedtwitz, P. V., Osinga, T., Kinamore, J., and Steinfeld, A., 2003 "A New $75 \mathrm{~kW}$ High-Flux Solar Simulator for High-Temperature Thermal and Thermochemical Research," ASME J. Sol. Energy Eng., 125(1), pp. 117-120.

[12] Petrasch, J., Coray, P., Meier, A., Brack, M., Häberling, P., Wuillemin, D., and Steinfeld, A., 2007, "A Novel 50 kW 11,000 Suns High-Flux Solar Simulator Based on an Array of Xenon Arc Lamps," ASME J. Sol. Energy Eng., 129(4), pp. 405-411.

[13] Dibowski, H.-G., 2014, "High-Flux Solar Furnace and Xenon-High-Flux Solar Simulator," accessed: Mar 28 2014, http://www.dlr.de/sf/en/desktopdefault.aspx/ tabid-8558/14717_read-28267

[14] Krueger, K. R., Davidson, J. H., and Lipiński, W., 2011, "Design of a New $45 \mathrm{kWe}$ High-Flux Solar Simulator for High-Temperature Solar Thermal and Thermochemical Research," ASME J. Sol. Energy Eng., 133(1), p. 011013.

[15] Krueger, K. R., Lipiński, W., and Davidson, J. H., 2013, "Operational Performance of the University of Minnesota 45 kWe High-Flux Solar Simulator," ASME J. Sol. Energy Eng., 135(4), p. 044501.

[16] Sarwar, J., Georgakis, G., LaChance, R., and Ozalp, N., 2014, "Description and Characterization of an Adjustable Flux Solar Simulator for Solar Thermal, Thermochemical and Photovoltaic Applications," Sol. Energy, 100, pp. 179-194.

[17] Osram, Manufacturer Data.

[18] NREL, "Reference Solar Spectral Irradiance: Air Mass 1.5," accessed July 10 2014, http://rredc.nrel.gov/solar/spectra/am1.5/ 
[19] Siegel, R., and Howell, J. R., 2002, Thermal Radiation Heat Transfer, Taylor \& Francis, NY.

[20] Osram, XBO® Theater Lamps, Technology and Application.

[21] Rabl, A., 1985, Active Solar Collectors and Their Applications, Oxford University, NY.

[22] Duffie, J. A., and Beckman, W. A., 2006, Active Solar Collectors and Their Applications, Wiley, Hoboken, NJ

[23] Krueger, K. R., 2012, "Design and Characterization of a Concentrating Solar Simulator," Ph.D. thesis, University of Minnesota Minneapolis, MN.
[24] Petrasch, J., 2010, "A Free and Open Source Monte Carlo Ray Tracing Program for Concentrating Solar Energy Research,” ASME Paper No. ES201090206.

[25] Johnston, G., 1995, "On the Analysis of Surface Error Distributions on Concentrated Solar Collectors," ASME J. Sol. Energy Eng., 117(4), pp. 294-296.

[26] Hischier, I., Poživil, P., and Steinfeld, A., 2012, "A Modular Ceramic CavityReceiver for High-Temperature High-Concentration Solar Applications," ASME J. Sol. Energy Eng., 134(1), p. 011004. 